\title{
Spinal Body Replacement and Endoscopic Reconstruction
}

\author{
Stefan Rose', Ingo Marzi²
}

Fractures, tumors, and bacterial infections of the thoraco-lumbar spine often require dorsal stabilization to reconstruct the vertebral alignment or to support tension band stability. In many cases, and especially in fractures, we have learned that healing of the injured spinal segment is not in any case accomplished by dorsal procedures alone. Since the stability of the whole spinal segment mainly depends on the integrity of the ventral load-bearing column, we should consider anatomic reconstruction of the vertebral body when indicated. Since main secondary loss of correction derives also from the injured disc space [1], disc replacement is a further indication for ventral spinal stabilization. In this respect, MRI has a high diagnostic relevance and might help in decision-making using mono- or bisegmental ventral spondylodesis.

Although we have gathered a lot of experience with different stabilization devices and products, neither the definite concept performing spinal stabilization, nor perfect implants, biological or artificial, are yet available. A prospective multi-center study including 682 patients comparing internal fixateurs combined with bone grafts, dorso-ventral, and isolated ventral stabilizations demonstrated in one third of the patients' functional impairment, for all techniques a loss of reduction and especially a high donor-side morbidity of the tricortical bone graft. The study, however, concluded that the combined dorsoventral fusion brings about the best reduction over time [2].

Some questions have to be asked. How much stabilization is necessary? How to reconstruct: with bone,

Eur J Trauma 2006; 32: 213-214

DOI 10.1007/s00068-006-9306-9

\footnotetext{
Groupe Chirurgical Ettelbruck, Centre Hospitalier du Nord, Hopital St. Louis, Ettelbruck, Luxembourg

2 Department of Trauma, Hand, and Reconstructive Surgery, University Hospital, Johann Wolfgang Goethe University, Frankfurt/Main, Germany
}

plate, or cages? What to do in situations like infection and severe osteoporosis? How to approach mono- and bisegmental injury patterns? What about the risks of ventral stabilization procedures?

In this issue, we published a series of papers dealing with the anterior spinal body reconstruction after trauma, tumors, and infection. The papers critically evaluate surgical procedures, drawbacks, and pitfalls that will be very helpful for spine surgeons of all disciplines. In order to discuss these papers and open questions thoroughly, we want to put forward some basic considerations, which may be kept in mind during reading:

Issue I. Independently of the surgical approach (endoscopic, mini-open, open) one must first analyze the fracture type in order to choose the right stabilization concept. The rising numbers of ventral procedures have the risk that the dorsal tension band provided by the internal fixateur might be underestimated as a stabilization concept. Due to better implants, one can observe increasing considerations and a tendency to approach instable AO type A and B fractures just from the front. So far, available constructs might not be able to support the ventral column with concomitant dorsal instability. The (AO) classification of Magerl et al. [3], which counts for injury mechanisms like compression, distraction, and rotation, provides a useful tool to safely treat thoraco-lumbar fractures. Although, it might be difficult to correctly define the different subgroups, one should be quite decisive for $\mathrm{AO}$ type $\mathrm{A}, \mathrm{B}$, or $\mathrm{C}$ fracture pattern before any treatment. Isolated ventral stabilizations (rare, ca. 10\%) should only performed in AO type A1 fractures with 
persistent pain and AO type A2-3 fractures. As implants, one can use plate systems (angular stable) with tricortical bone grafts or titan cages with spongy bone. However, the latter should be used very carefully as a stand-alone implant. All other fractures might be primarily stabilized by a dorsal internal fixateur, followed by ventral reconstruction (Figure 1).

Issue II. There is still debate how to treat monosegmental injuries. Monosegmental stabilizations, even performed with cages, are possible in incomplete compression and burst fractures with intact caudal disc, stable caudal spinal body, and protected by angular stable osteosynthesis. However, in patients with impaired bone quality and insufficient protection of the ventralimplants, subsidence of the implants is a major concern and might cause technical problems. This issue needs to be further addressed, especially in elderly patients and osteoporosis.

Issue III. A failure of ventral implants is closely related to the right surgical indication, the right surgical approach, the right surgical technique, and the right choice of implant. We see failure of ventral implantations which cannot at all be attributed to the used implant itself. In many cases, underestimated fracture patterns and technical problems can be analyzed. However, the question whether bone grafts alone or protected by plates, or titan cages might be the better choice is not yet scientifically decided. So far, several highly-developed plate and cage systems suitable for minimal-invasive implantation are available which have to be investigated in terms of long-term stability, subsidence, and healing of the injured spinal segment. Titan cages might be a suitable implant to treat tumors and infections, in the latter case combined with a thorough debridement. Moreover, we have to discuss donor site morbidity when using pelvic rim or rib grafts. In this respect, a future goal should be the development of artificial and biological bone substitutes for spinal body replacement.

Issue IV. Introduction and development of endoscopic spine surgery has widely increased the number of ventral stabilizations. Video-imaging and thoracoscopic instruments were first developed and used by cardio- and thoracic surgeons [4]. Conventional thoracotomies have
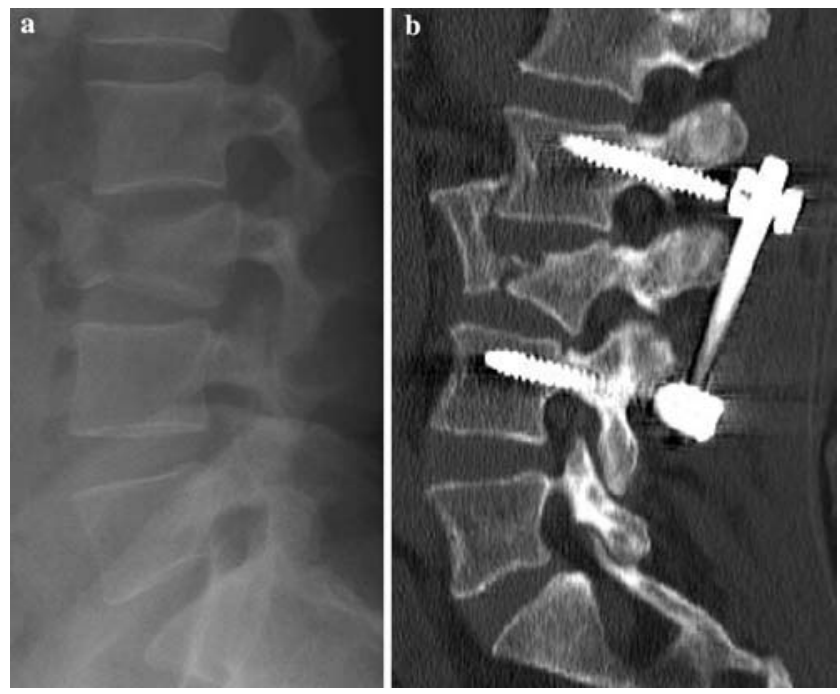

Figure 1. Failure of dorsal spondylodesis by internal fixateur due to insufficient support of the ventral column in AO type B fracture of $L_{3}$.

shown complications like intercostal neuralgia, post thoracotomy syndrome, hemiparesis of the diaphragm, and the drawback of a large approach for a 'small' injured area. In contrast, several studies could show that minimal-invasive endoscopic procedures reduced postoperative pain, speeded postoperative rehabilitation, reduced hospital stay, pulmonary complications and shoulder girdle, and approach-related morbidity $[5,6]$. We clearly need for endoscopic procedures a thorough preoperative planning and we have to respect the specific risk factors of thoracic surgery. Nonetheless, the use of endoscopic and minimal-invasive approaches for spinal body replacement is an appropriate and safe method.

\section{References}

1. Eysel P, Rompe JD, Hopf C, et al. Significance of the intervertebral disk in failed reduction of surgically stabilized fractures of the truncal spine. Unfallchirurg 1994;97:451-7.

2. Knop C, Blauth M, Bühren V, et al. Surgical treatment of injuries of the thoracolumbar transition-3: follow-up examination. Results of a prospective multi-center study by the "Spinal" Study Group of the German Society of Trauma Surgery. Unfallchirurg 2001;104:583-600.

3. Magerl A, Aebi M, Gertzbein SD, et al. A comprehensive classification of thoracic and lumbar injuries. Eur Spine J 1994;3:184-201.

4. Mack MJ, Regan JJ, Bobechko WP, et al. Application of thoracoscopy for diseases of the spine. Ann Thorac Surg 1993;56:736-8.

5. Hertlein $\mathrm{H}, \mathrm{Hartl} \mathrm{WH}$, Dienemann $\mathrm{H}$, et al. Thoracoscopic repair of thoracic spine trauma. Eur Spine J 1995;4:302-7.

6. Bühren V, Beisse R, Potulski M. Minimally invasive ventral spondylodesis in injuries to the thoracic and lumbar spine. Chirurg 1997;68:1076-84. 